\title{
BALANCED SCORECARD COMO HERRAMIENTA PARA EMPRESAS EN ESTADO DE CRISIS
}

\author{
The balanced scorecard as a tool for companies in crisis
}

\author{
Lewis Charles Quintero-Beltrán \\ MSc en Administración. Escuela de Economía, Administración y Negocios, \\ Universidad Pontificia Bolivariana, Medellín-Colombia, lewis.quintero@upb.edu.co
}

\section{Licet María Osorio-Morales}

MSc en Administración. Escuela de Economía, Administración y Negocios, Universidad Pontificia Bolivariana, Medellín-Colombia, licetosorio@hotmail.com

\begin{abstract}
Cómo citar / How to cite
Quintero-Beltrán, L., \& Osorio-Morales, L. (2018). Balanced scorecard como herramienta para empresas en estado de crisis. Revista CEA, 4(8), 75-94. https://doi.org/10.22430/24223182.1049
\end{abstract}

Recibido: 15 de febrero de 2018

Aceptado: 20 de mayo de 2018

\section{Resumen}

Este artículo tiene el propósito de analizar la pertinencia del Balanced Scorecard (BSC) en empresas en crisis, mediante una exploración documental que posibilite comprender las oportunidades que brinda esta herramienta para la gestión organizacional. Así, se parte de la hipótesis de que efectivamente el BSC es de suma utilidad en las empresas y su implementación tiene un efecto positivo, por lo que inclusive su aplicación puede llegar a ser más una necesidad imperativa que una elección transigente. De esta manera, se realiza un recorrido histórico sobre el BSC y su nacimiento en Estado Unidos, se efectúa una investigación documental en la que se resaltan algunos ejemplos de aplicación del modelo del BSC, y finalmente se desarrolla una comparación entre este modelo y su implementación en empresas que enfrentan crisis organizacional, para sugerir una serie de recomendaciones en torno al uso del BSC en la estructura interna de las compañías.
Palabras clave: Balanced Scorecard, crisis organizacional, gestión organizacional, exploración documental.

\begin{abstract}
This article aims to analyze the relevance of the Balanced Scorecard (BSC) for companies that face periods of crisis by means of a literature review that enables to understand the opportunities this tool offers for organizational management. Thus, this work is based on the hypothesis that the BSC is indeed extremely useful for companies and its implementation has a positive effect; hence, its application may be more an imperative than a choice. The history of the BSC and its birth in the United States are also analyzed. Furthermore, this review highlights some real examples of the implementation of the BSC model. Finally, a comparison is drawn between such model and its actual implementation in companies that experience an organizational crisis, in order to suggest a series of recommendations on the use of the BSC in the internal structure of companies.
\end{abstract}


Keywords: Balanced Scorecard, crisis management, organizational management, literature review.

\section{INTRODUCCIÓN}

Las nuevas dinámicas del mercado, que surgen a partir de la llegada de la globalización y la apertura de nuevas formas de comercio, resultado de las políticas liberales en el mercado, llevan a formular la necesidad de nuevas herramientas de gestión y control de una empresa. En este contexto surge la creación del BSC (Balanced Scorecard), instaurada a finales del siglo XX por Norton y Kaplan.

El problema central que trabaja la siguiente investigación se basa en las empresas que están en crisis organizacional, ya que en estas no hay una herramienta que garantice medir, evaluar y conocer los errores o debilidades de una agrupación. De esta manera se hace necesario establecer una relación directa del BSC, con las empresas en crisis organizacional. Si bien la situación de estas compañías no es algo nuevo en lo referente a las mediciones y su impacto en la eficiencia, es un fenómeno que se ha acentuado en los últimos años, dada la enorme proporción de nuevas empresas que emergen en distintos momentos, lo que acrecienta las prácticas de competencia y hace más difícil la subsistencia de estas en el mercado, pero además, si realmente la empresa cumple sus objetivos, resulta primordial cuestionarse ¿de qué manera el Balanced Scorecard posibilita una oportuna gestión en empresas con crisis organizacional? En este sentido, se justifica la conveniencia de este documento, ya que su aplicación y resultados pueden llegar a ser de gran utilidad en empresas que necesiten una guía para superar o evitar la crisis organizacional. Sin embargo, el aporte se plantea también para la comunidad de profesionales dentro de áreas de administración y gestión de empresas, dedicados a gerencia y finanzas de administración o carreras y profesiones afines, que busquen aplicar la técnica del BSC, en alguna compañía.

En consecuencia, el objetivo de la investigación es analizar la pertinencia del uso de la herramienta BSC en empresas con estado de crisis organizacional, para lo cual en primer lugar se realizó una exploración documental que posibilitó comprender las oportunidades que brinda esta herramienta para la gestión organizacional, teniendo como referente empresas que han tenido éxito en la implementación de la herramienta y otras que no han logrado migrar a la utilización del BSC; en segundo lugar, se identificaron prácticas concretas de empresas que han implementado el modelo del BSC; y en tercer lugar, a partir de la recopilación documental, se examinaron beneficios concretos de la aplicación del modelo BSC para las empresas en crisis y se desglosaron una serie de recomendaciones $y$ conclusiones.

La presente investigación es de tipo documental, por lo tanto, se realizó una exploración y revisión de distintos autores que definen, combinan e integran el Balanced Scorecard como un modelo de gestión empresarial. Así mismo, esta investigación es de carácter cualitativo, pues hay una necesidad de evaluar la pertinencia del BSC en empresas en crisis y la pertinencia de este modelo para las compañías que cuentan con una mala gestión organizacional.

Por ende, en primera instancia se realizó una revisión de documentos fruto de consultoría en empresas que tratan acerca del Balanced Scorecard y que dan muestra de sus beneficios y dificultades dentro de una empresa, sin importar el sector. Es decir, se mostró un panorama lleno de posibilidades, donde los documentos han evidenciado contrastes históricos, contextuales e institucionales que legitiman la pertinencia del Balanced Scorecard. 
Luego, se seleccionó algunos documentos a partir de los cuales se estableció un diálogo continuo que permitió develar elementos claves para el artículo, en tanto, el Balanced Scorecard podrá ser la estrategia pertinente para las empresas en crisis organizacional. Para lo que se tomaron en cuenta los casos del Grupo Santillana Ecuador, el caso de QUIPUX S.A.S y el caso de JAB S.A.S.

Finalmente, se sistematizó y se mostraron los avances, transformaciones y beneficios de la aplicación del Balanced Scorecard en empresas en estado de crisis, así, se llegó a una serie de conclusiones que permiten establecer una conciencia y postura reflexiva frente al uso de esta herramienta en diversas organizaciones. Dado que la investigación se realiza en torno a la aplicación de BSC como herramienta para superar el estado de crisis en organizaciones, se parte de la posibilidad de que el BSC es de suma utilidad en las empresas y su implementación tiene un efecto positivo, por lo que inclusive su aplicación puede llegar a ser más una necesidad imperativa que una elección transigente.

\section{MARCO TEÓRICO: EL BSC (BALANCED SCORECARD), APROXIMACIONES A SUS ANTECEDENTES HISTÓRICOS}

Los 80 fueron sin duda un periodo de crisis financiera en Estado Unidos, pues con la llegada de Reagan a la presidencia el país sufrió un proceso de recesión. Este estado de recesión se establecía para lograr enfrentar y disminuir los altos índices de inflación que dejaron las administraciones políticas pasadas.

Aunque esta forma política y económica de sacar al país de su crisis financiera logró estabilizar por un tiempo la economía norteamericana, tuvo mayores consecuencias. Entre muchas otras, a nivel social, aumentó el índice de pobreza que afectó a grupos poblacionales de minorías étnicas, los sistemas de protección social y el sistema empresarial. A nivel económico, este periodo de recesión estaba involucrado en los inicios de la apertura y establecimiento de las políticas liberales en la economía mundial, lo cual en consecuencia establecía nuevos ritmos en el mercado, la oferta, la demanda y también en la forma de competencia.

Así mismo, estos modelos de política liberal impactaron algunas regiones de Latinoamérica, abriendo paso a instaurar los mismos modelos liberales dentro de su estructura política y económica, estableciendo elementos de libre competencia y dejando de lado el papel regulatorio del Estado en la actividad económica del país, como lo explica Bouzas (1982).

En este sentido, debido a los periodos de cambios que se experimentaban a nivel mundial con la instauración de modelos liberales, Kaplan y Norton (1996) entendieron que las empresas tenían sistemas que no se ajustaban a las demandas del mercado, por lo que diseñaron el modelo del BSC (Balanced Scoredcard) como una herramienta que permitiera que una empresa llegara a generar una gestión eficaz, un plan de gerencia $y$ evaluación de proyectos, que de forma tangible lograra entender las dinámicas de la empresa frente a las necesidades del mercado.

Al observar el contexto histórico en el que se crea el BSC, es posible entender que las empresas enfrentaban un proceso de cambio, es decir, unos nuevos ritmos económicos inmersos en las nuevas políticas liberales. De hecho, Kaplan y Norton introdujeron el BSC a finales del siglo XX, por la insuficiencia de los medidores financieros en las empresas norteamericanas de la época.

Al respecto, Kaplan y Norton (1996) explican que la competencia empresarial ha sufrido diversas transformaciones, en donde, por ejemplo, si se ubican en el periodo de la Revolución Industrial, la competencia únicamente se deducía desde la capacidad de 
adaptación a la tecnología y la capacidad de adquisición de la maquinaria o herramientas de trabajo, hasta la producción en masa y de calidad, estos eran los factores principales para garantizar la sostenibilidad de una empresa. Este primer enfoque se media de acuerdo con las valoraciones de la contabilidad financiera.

De manera que, en la nueva era de la información, la competencia se entiende como un proceso y actividad, en el que una empresa entiende que tanto los activos físicos y tangibles como los intangibles son factores que permiten que una organización desarrolle diversas características, que le permitan tener un nivel de competencia y estabilidad en la era de la información.

Para responder a esos cambios económicos y de mercado a nivel empresarial, Kaplan y Norton (1996) realizan una investigación, en la cual diseñan un modelo titulado BSC Balanced scorecard o cuadro de mando integral, el cual permite a una empresa adquirir la cualidad de adaptación en torno a diferentes cambios que se presentan en los diferentes sectores empresariales, entendiendo que el cambio es una constante inherente a la organización. Para desarrollar esta cualidad y encontrar una estabilidad, las empresas deben fijar visiones y objetivos a largo plazo y formular estrategias que deben cumplirse y ser realizables, por lo que sus resultados son tangibles y medibles a través de unos indicadores. Esta investigación realizada por Norton y Kaplan la aplican a 12 empresas, las cuales se reunieron bimestralmente en 1990 para generar un nuevo modelo de medición de la actuación. En este ejercicio Kaplan y Norton logran llevar a la realidad la teoría y satisfactoriamente establecer el modelo del BSC en los procesos internos de gestión de las empresas.

Ahora bien, el BSC básicamente surge a partir de la planeación estratégica que "consiste en un ejercicio de formulación y establecimiento de objetivos de carácter prioritario, cuya característica principal es el establecimiento de los cursos de acción estrategias para alcanzar dichos objetivos» (Armijo, 2009, p.5).

En este sentido, una de las mayores dificultades que presentaban las empresas a la hora de realizar la planeación estratégica era efectuar la implementación con éxito. En consecuencia, Kaplan y Norton (1996) crearon el BSC como una herramienta para otorgarle a una empresa la forma de implementar sus estrategias, es decir, que el BSC ayudar a resolver ese paso de la formulación del proyecto a la ejecución de la estrategia.

El BSC es un modelo de gestión que ayuda a una empresa a llegar a cumplir su visión, a través de la ejecución de unos objetivos, los cuales están enfocados sobre todo en la creación de valor. Es decir, que es la herramienta base que ayuda a generar la sostenibilidad de la empresa, a través de una serie de discusiones gerenciales y de formulación de proyectos del equipo directivo, acerca del funcionamiento del negocio, con el objetivo de efectuar y establecer diversas prioridades de la empresa frente al mercado. En este sentido, el BSC es una herramienta que a partir del énfasis en la creación de indicadores financieros $e$ indicadores no financieros, transforma las estrategias y los objetivos de un negocio en indicadores tangibles.

De esta forma, es posible entender que su nombre BSC se refiere al equilibrio que una organización puede obtener entre sus objetivos, sus medidas financieras y no financieras, sus indicadores y su actuación interna y externa, proporcionando una estructura para un sistema de gestión y medición estratégico, el cual a nivel de ejecución comprende diversas etapas, como la implementación de un estudio previo de la empresa y el mercado, la formulación de grupos de trabajo dentro de los diferentes departamentos de la empresa, para así llegar a la creación de la primera fase del BSC que es la creación del mapa estratégico. Este mapa 
integra 4 perspectivas: la financiera; la del cliente; la interna; y la innovación formación (ver Tabla 1).

Con base en el mapa estratégico, se elabora la segunda fase del BSC, que es la tabla de control en la cual se añaden objetivos estratégicos con cada una de las
4 dimensiones. También unos indicadores de desempeño para medir los objetivos y unas iniciativas estratégicas; cada una de estas variables deben cumplir con las características de ser específicas, medibles, alcanzables, relevantes, y deben cumplirse en un determinado tiempo. Ver Tabla 2.

Tabla 1. Mapa estratégico del BSC

Table 1. Strategy map of the BSC

Perspectiva financiera

Perspectiva comercial

Perspectiva procesos internos

Perspectiva aprendizaje y desarrollo
Se refiere al incremento de ingresos que una empresa espera obtener, usualmente en esta perspectiva se ubica el alza o baja de costos y el aumento de ventas.

Se refiere a la forma en que se pretende satisfacer a un cliente y retener para que se fidelice con la empresa, o también las estrategias para elevar el número de clientes.

Se refiere a las estrategias en los procesos internos de la empresa, en su estructura organizacional, como la generación de operaciones de gestión de clientes, de innovación, de medio ambiente-comunidad.

Aquí se observan las competencias que debe mejorar una empresa, sobre todo en cuanto al capital humano, que pueden generar estrategias de motivación, capacitación, mejorar de tecnologías etc.

Fuente: elaboración propia, 2017.

Tabla 2. Ejemplo de Tabla de Control

Table 2. Example of a control table

\begin{tabular}{l|l|l|l|l|l}
\hline & Objetivos & Indicadores & Metas & $\begin{array}{l}\text { Iniciativas o variables } \\
\text { de acción }\end{array}$ & Responsables \\
\hline $\begin{array}{l}\text { Perspectiva } \\
\text { financiera }\end{array}$ & & & & & \\
$\begin{array}{l}\text { Perspectiva de } \\
\text { cliente }\end{array}$ & & & & & \\
Perspectiva interna & & & & & \\
$\begin{array}{l}\text { Perspectiva de } \\
\text { aprendizaje }\end{array}$ & & & & & \\
\hline
\end{tabular}


Varios autores han añadido nuevas definiciones sobre lo que significa, sus funciones y usos en diversas empresas. Uno de estos es Fernández (2001), quien define el BSC como un modelo de gestión, el cual, a partir de la medición de indicadores de objetivos ligados a un plan de acción, permite el control y la lectura de los comportamientos de una organización. Es decir que el BSC podría ser una herramienta que permite examinar las acciones de una empresa a corto, mediano y largo plazo.

Así mismo, el BSC tiene una serie de elementos que corresponden a la implementación de una misión, visión y valores, la construcción de unos mapas estratégicos, perspectivas y objetivos, iniciativas estratégicas, responsables y recursos; y finalmente, el elemento de la evaluación y retroalimentación.

Otros autores que reflexionan sobre el BSC son Trullenque y Linquete (2002), Malgioglio (2002), Neriz (2006) y Mañas (2005), entre otros, los cuales definen el BSC muy similarmente, variando en las ventajas $y$ necesidades de una empresa al implementar el tablero de mando balanceado. Entre ellas se destacan las ventajas de establecer causaefecto, establecer inductores de actuación y la vinculación con las finanzas, así mismo mejorar las estrategias de organización. También es vista como una herramienta de control que permite medir y evaluar procesos internos y se puede relacionar con el proceso de cascada o también con la herramienta de DOFA, encaminando la empresa siempre hacia una perspectiva de éxito a través de unas metas que se implementan en base al concepto de excelencia.

En Colombia también se ha trabajado e implementado el modelo del BSC dentro de las empresas privadas, las empresas públicas y las instituciones estatales. En este sentido, la Alcaldía de Bogotá (2007) realizó una guía para la elaboración del BSC, la cual fue implementada por la necesidad de fortalecer las empresas privadas como las empresas públicas, dentro de los procesos de gestión, control y medición, con el objetivo de fomentar el desarrollo institucional en el distrito capital. Esto demuestra el impacto que ha tenido el BSC en las organizaciones, puesto que incluso su uso se ha desarrollado en entornos políticos; es decir, que es una herramienta que ha resultado de utilidad en el ámbito de evaluación y gestión de proyectos.

\section{La crisis: sobre empresas en crisis organizacional}

Ahora bien, el artículo se basa en el estudio del BSC en las empresas que se ubican en un periodo de crisis organizacional. En este sentido, la crisis se trabaja desde la perspectiva de Coombs (2014), quien define una crisis como una amenaza significativa para las operaciones y reputaciones de una empresa, la cual puede tener consecuencias negativas si no se maneja adecuadamente. En la crisis organizacional, la amenaza es el daño potencial que una crisis puede infligir a una organización, sus grupos de interés y su industria. Una crisis puede crear amenazas de seguridad pública, generar una pérdida financiera, puede estar dirigida a afectar la imagen y reputación de una empresa o afectar las tres dimensiones. Estas amenazas señaladas usualmente están interconectadas, es decir, que el manejo erróneo de una puede afectar de la misma forma a las otras.

Así mismo, la crisis se puede observar como un evento de alto impacto que puede ser negativo, e inclusive se define como un proceso que puede generar la aniquilación de una organización. Ninguna empresa está a salvo o exenta de un evento de crisis, por lo que es importante realizar procesos, bien sea de previsión y contención, para prepararse para sucesos que puedan generar diferentes 
deterioros en la estructura de la organización. "Las organizaciones preparadas para manejar las crisis limitan hasta a un $30 \%$ el impacto previsible y se recuperan hasta el doble de rápido que las que no lo están» (Remy, 2013, p. 3).

Una crisis puede presentarse tanto por factores externos, como por los cambios de mercado, la competencia, la relación con el cliente; también por factores internos, como relaciones con los empleados, operatividad, adecuación de un espacio favorable de trabajo, insumos laborales propicios, clima organizacional, entre otros. Es importante entender que la crisis representa una serie de etapas que cualquier empresa puede llegar a vivir, por lo que, a mayor proceso de anticipación, menor es la intensidad en la que se siente la crisis; mientras que, a menor preparación de anticipación, la crisis desemboca con un mayor y alto impacto.

Para prever una crisis hay herramientas como el mapa de riesgos, el cual permite a través de una comunicación efectiva entre los departamentos de las empresas generar un panorama sobre las áreas que presentan mayores riesgos, y sobre posibles colapsos (Remy, 2013). Si una empresa se ubica en estado de crisis organizacional es importante que sus directivos tengan una reacción rápida y asertiva para no dejar crecer en mayores proporciones un problema, que puede encontrar un desenlace en la liquidación de una compañía. Esto exige un grado de conciencia alto en los directivos de la organización, quienes deben aceptar el proceso al que se enfrenta y ocuparse de este en el menor tiempo posible.

En este sentido, la comunicación es un eje fundamental, puesto que puede ser un arma de doble filo, ya que si no se maneja de una forma adecuada puede generar imágenes erróneas de la compañía y producir caos en los empleados. Mientras que si se maneja de una forma correcta puede llegar a amortiguar el golpe de la crisis, tanto de manera interna como de manera externa.

La recuperación de una empresa en estado de crisis, explica Remy (2013), genera un proceso de aprendizaje, el cual permite dimensionar los alcances de la organización en diversos ámbitos, así como también los alcances individuales de cada empleado. En esta etapa de recuperación es preciso que una empresa haga una pausa para entender ¿cuál fue el proceso al que se enfrentó? Y ¿qué riquezas puede sacar de este proceso para fortalecer la compañía?

\section{ANÁLISIS Y RESULTADOS: DE LA CRISIS AL ÉXITO, CASOS TRIUNFANTES DEL BSC}

Para analizar la pertinencia del Balanced Scorecard en empresas en crisis, mediante una exploración documental que posibilite comprender las oportunidades que brinda esta herramienta para la gestión organizacional. A continuación, se presenta el diálogo entre los textos revisados en los cuales el Balanced Scorecard se presenta como la estrategia pertinente para las empresas en crisis organizacional.

EI BSC es una herramienta de control de una empresa, que permite una lectura de la ejecución de estrategias para llegar a una meta e inclusive puede funcionar como un escudo preventorio de crisis, como se mencionó anteriormente. En este sentido, las empresas como Kimberly Clark y Exxonmobil, fueron las pioneras en Colombia en instaurar el modelo de BSC en su compañía, con el objetivo de fortalecer sus estructuras internas. Otro caso célebre fue el proceso del Banco de Crédito, compañía que por medio del uso del BSC salió de una crisis: «El Banco de Crédito lo utilizó inicialmente para recuperar la rentabilidad en medio de las condiciones adversas de la 
recesión y la crisis del sistema financiero entre 1999 y 2000» (Dinero, 2005). Así es posible observar que el BSC ha sido una herramienta que han usado empresas, tanto en crisis, como empresas con éxito, para seguir liderando un mercado.

De la misma forma, empresas reconocidas como Schilinder, Telefónica Citibank e Hidrocamtábrico han adoptado el modelo del BSC dentro de sus estructuras y procesos de gestión empresarial. Lo han realizado con el objetivo de formar esas estrategias de control, que les permitan posicionar la empresa, mantener una relación efectiva frente a los clientes o superar las crisis generadas por las dinámicas del mercado global. Cabe resaltar que el BSC es un proceso interno de una empresa. Por lo tanto, rara vez son públicos, puesto que hacen parte un proceso que se ubica dentro del secreto empresarial. Es decir, no son accesibles al público en general, porque en ellos inclusive puede encontrarse un elemento de ventaja de una empresa frente a la competencia. En referencia a lo anterior Ortiz-Delgadillo, G., Esquivel-Aguilar, E. O., \& HernándezCastorena, O. (2016) mencionan que el entorno económico actual se caracteriza por la creciente complejidad de las organizaciones y su entorno, en las cuales el constante cambio en los gustos y hábitos de los consumidores ha producido un desequilibrio en la demanda de los productos ofrecidos, donde factores como la innovación, la competitividad y la calidad han marcado tendencia sobre la dirección estratégica que siguen las empresas en busca de satisfacer las nuevas expectativas del mercado.

Sin embargo, hay casos que se pueden explorar, por ejemplo, es fácil encontrar documentos relacionados al BSC en empresas públicas o distritales. También en la investigación fue posible encontrar ejemplos reales de la aplicación del BSC en algunas empresas, pues fueron resultado de un análisis y por lo tanto su publicación se divulgó dentro de este ámbito.

De estos ejemplos se recurrió a tres casos concretos, puesto que su elaboración estudio y relato, dentro de la investigación, resultaron ser los más complejos y detallados. Lo que interesa en el artículo es en esencia poder observar detalladamente la implementación de esta herramienta y el éxito en los resultados implementado en empresas en crisis, así como las adversidades a las que se enfrentaron. Lo siguiente se realizó con el objetivo de analizar prácticas concretas de empresas que han implementado el BSC, con la finalidad de comprender la pertinencia de dicho modelo para las compañías de distintos sectores que se encuentren en crisis organizacional.

En este sentido, el primer caso que llamó la atención en el proceso de investigación fue el del Grupo Santillana Ecuador. Este caso lo realizó Vogel (2011), quien formuló con éxito el uso de la herramienta del BSC para la empresa. Así mismo en el documento se realizan una serie de reflexiones en torno a los aspectos necesarios para generar una implementación exitosa del BSC, a partir de observar las dificultades y las ventajas que forjó este proceso en la estructura interna de la organización.

En la investigación de Vogel (2011), se logró observar que parte de un antecedente histórico, es decir un estudio previo sobre la empresa. A partir de esta base se realizó la implementación del BSC en el 2009, en el Grupo Santillana, el cual forjó el BSC desde la perspectiva en la cual se ve como herramienta de control, para mejorar la lectura en cuanto al desempeño financiero de la empresa y las estrategias para mantenerse posicionado en el mercado. Para su implementación, la empresa realizó un diagnóstico de la situación actual, formuló unas estrategias, objetivos, indicadores, metas, iniciativas, planes de 
acción, evaluaciones y retroalimentaciones. El primer paso consistió en realizar un mapa estratégico.

Después de realizar el mapa estratégico, se pasó a definir los objetivos estratégicos de la corporación, siguiendo los pasos regulares descritos por Kaplan y Norton (1996) de BSC. Esta etapa consistió en apoyarse en el mapa para generar a cada perspectiva sus correspondientes objetivos. Los cuales a su vez surgen de preguntas en cuanto a necesidades, carencias y percepciones; estas preguntas pueden ser transversales a cada paso, es decir, en la formulación de objetivos de cada perspectiva.

El siguiente paso consistió en la identificación del personal, la información y los procesos comunicativos como elementos vitales en la toma de decisiones. También, se asignaron los indicadores, se establecieron las metas a mediano y largo plazo, se realizó una matriz que permitiera identificar cuáles son las iniciativas y su grado de prioridad. A partir de lo anterior, se establecieron umbrales porcentuales que permitieron medir el rendimiento por medio de un parámetro de semáforos, es decir $60 \%$ o menos rojo, de $61 \%$ a $80 \%$ amarillo y superior a $80 \%$ verde. Al finalizar esta etapa se procedió a formar el cuadro de mando integral.

En este caso, se observó que las principales dificultades para la implementación del modelo del BSC, se ubicaron dentro de los procesos comunicativos, pues por ser una empresa que maneja múltiples áreas y gran número de personal, es difícil generar consensos en cuanto a definir los objetivos y componentes que debe cumplir cada área de la empresa. Así mismo, se logró comprender que involucrar todas las áreas de la organización para generar un trabajo en equipo también fue difícil, puesto que en el modo organizacional de la compañía el trabajo individual era el que primaba.

Otro inconveniente fue el modelo de liderazgo, puesto que cada jefe de departamento debe incentivar la implementación de estrategias para lograr cumplir con las metas por área. En consecuencia, cada líder de cada área tiene un papel principal dentro de la comunicación en la organización.

También se ubicaron fallas en la falta de enfoque de la compañía, debido a que no se tenía una delimitación en cuanto al mercado al cual se iba a dirigir, es decir, que no se tenía una definición clara sobre los alcances de mercado de la empresa. Por otro lado, las múltiples opiniones de cada sector, el personal rotativo, poca cultura gerencial en el uso de indicadores, metas y rubros financieros, fueron algunas de las dificultades que se presentaron en el momento de instaurar el modelo.

Ahora bien, dentro de los beneficios que obtuvo la empresa al instaurar el BSC, se ubicaron los relacionados a la reducción de costos, reestructuración de algunos procesos internos favorables, agilidad en procesos administrativos, estructuración de una atención adecuada hacia el cliente, toma de decisiones asertivas en torno al enfoque $y$ metas de la organización.

Así mismo, la comunicación con el personal mejoró mediante la escucha activa a diversas propuestas. Es interesante en este caso observar que se formuló una cultura frente al BSC, puesto que según Vogel (2011), la empresa logró establecer un estado de conciencia, el cual entiende que el BSC es un proceso continuo que debe estar en constante reestructuración y adaptación. Es decir, que no es estático por lo que siempre debe estar funcionado y en proceso de cambió. 
Esta cultura creada por el BSC se puede ubicar dentro de la estructura interna de la empresa, en diversos espacios como el desarrollo de actitudes proactivas, cultura de medición y cultura de evaluación entre otros. Estos aspectos permiten agilizar las reuniones y visualizar el estado de la empresa, a partir de la discusión de objetivos cumplidos o no, metas alcanzadas o no y las iniciativas que tienen un estado de prioridad. Según el informe, una de las ventajas que obtuvo el Grupo Santillana Ecuador, es que las discusiones que se llevan a cabo en las reuniones se establecen en un menor tiempo y con la característica de la fiabilidad. Puesto que cada una de estas discusiones se acompaña en la actualidad mediante indicadores de medición tangibles.

Otro ejemplo sobre la implementación del BSC es el realizado por Rincón (2013), el cual puso en funcionamiento del modelo del BSC en la empresa QUIPUX S.A.S., quienes tienen sede en Medellín Colombia y en Sao Paulo Brasil. Esta es una empresa desarrolladora de software, que presta servicios a sectores asociados con la movilidad. QUIPUX S.A.S tiene una característica notoria, pues ha estado en constante crecimiento desde su fundación en 1995. Este crecimiento, generó algunos vacíos en cuanto el auto conocimiento de la empresa y el control de varios procesos internos, por lo que para retomar el dominio fue necesario establecer técnicas de gestión y administración, que permitieran seguir fortaleciendo la estructura de la organización. Por este motivo la herramienta del BSC, se implementó a causa de la necesidad de la misma compañía.

De esta forma, para implementar el modelo del BSC en la empresa primero se observó la estructura organizacional; posteriormente a esto, se ubicó la misión y la visión, para lograr generar una definición de los objetivos estratégicos. Estos objetivos se ubicaron de acuerdo a su perspectiva es decir si era financiera, del cliente, interna o de innovación y aprendizaje. Así mismo se realizaron criterios de evaluación para priorizar tareas y actividades en base a los objetivos más importantes.

Seguido a esto se construyó el mapa estratégico y se establecieron criterios de éxito por eje, así como las medidas e indicadores para evaluar cada proceso. El mapa estratégico se realizó a su vez a partir de una serie de preguntas guías para establecer las medidas evaluativas Rincón (2013). Finalmente se pasó a observar las fuentes de información para que estas validaran las mediciones propuestas, es decir, que se realizó un diálogo con cada departamento para establecer un acuerdo frente a las unidades de medición.

Una vez que fue aprobado el modelo del BSC en la empresa, se pasó a su implementación y los resultados obtenidos contribuyeron a plasmar las estrategias de acción. De esta forma, ya la compañía no identificaba las estrategias como sueños o ideas en el aire, sino que se materializaron en acciones específicas para lograr metas concretas. Así mismo, se mejoró la comunicación interna en la empresa y a la vez se logró que varios actores concordaran con diversos conceptos y necesidades operativas en la empresa. Es decir que la materialización de las ideas fue uno de los logros del BSC, así como una reestructuración de las relaciones comunicacionales, de las evaluaciones, de los modelos de gestión, y de la participación de los integrantes, para generar una inclusión de varios sectores de la compañía.

Otro ejemplo es el de la empresa del sector industrial metalmecánico Laminados JAB S.A.S., en donde Grajales y Maldonado (2015) realizaron un diseño para implementar el modelo del BSC en la compañía. Llama la atención en este caso, que la empresa enfrentó un proceso de cambio en cuanto al sector del 
mercado al que va dirigido. En consecuencia, se estableció la necesidad de implementar el BSC en JAB S.A.S., puesto que la empresa para generar procesos de estabilidad, tuvo que instaurar un nuevo mercado y unos nuevos clientes a los que se dirigió. En este proyecto igual se empieza desde una contextualización de la empresa, ¿Cómo se organiza?, ¿Cuáles son sus principales funciones?, se estableció su marco legal, su marco histórico, la misión, la visión las políticas de calidad, funciones $y$ responsables. Estas características de evaluaron para generar un panorama que logre describir los principales problemas y así mimo formar un esquema de priorización de objetivos.

Este estudio realizó un proceso investigativo a fondo sobre la empresa, todos sus procesos, productos, el perfil de los empleados, entre otras cosas. A partir de este estudio se realizaron diversos diagnósticos que permitieron establecer las variables del BSC. Es posible observar una metodología rigurosa en este proyecto en cuanto a nivel de preinvestigación, es decir que a diferencia de los otros dos, Grajales y Maldonado (2005) realizaron un estudio a profundidad sobre el contexto histórico de la compañía y su actualidad.

Para aplicar el BSC se dividió el proyecto en 4 etapas, la primera fase correspondió a la identificación de la empresa en su totalidad para formular el mapa estratégico, la siguiente fase correspondió a definir los objetivos por cada uno de los 4 ejes del BSC. Luego en la tercera fase se procedió a establecer unos indicadores para identificar los resultados obtenidos en formas medibles y tangibles. Finalmente, en la última fase se procedió a realizar una autoevaluación del proceso.

Aunque en este proyecto se formuló en teoría el BSC es decir que no se aplicó, al final se realizaron algunas recomendaciones para lograr implementarlo con éxito dentro de la empresa. Estás se efectuaron en torno a la necesidad de establecer una participación activa en el personal de la compañía, así como la comunicación interna, la capacidad de toma de decisiones y consensos en el grupo de los directivos y también el compromiso de los líderes de cada departamento, puesto que ocupan una posición fundamental para la implementación del BSC de una forma exitosa.

Los anteriores casos, solo describen ejemplos del Balance Score Card. Es importante señalar que "no hay dos empresas iguales y tampoco dos implantaciones idénticas, las empresas han adaptado el modelo a sus propias necesidades o preferencias" (Fernández, 2001, p. 42). Es por ello que hay múltiples ejemplos en la red, de cómo es posible aplicar el modelo a una empresa. Realmente no es viable hablar de un método fijo, puesto que finalmente la implementación de este modelo, varía de acuerdo al tipo de mercado, cantidad de trabajadores, según la forma jurídica, su organización, su función, sus departamentos, objetivos entre otras características.

Por esta razón, los tres casos anteriores parten de necesidades diferentes para la instauración del modelo del BSC, dentro de sus estructuras. Así, la necesidad del Grupo Santillana Ecuador surge a partir de la exigencia de la editorial por generar una estabilidad en el mercado, es decir mantener su posicionamiento como editorial líder en el ámbito escolar en el Ecuador.

Por otro lado, el caso de QUIPUX S.A.S., surge a partir de la necesidad de la empresa de retomar el control y domino en los procesos internos, pues su constante crecimiento ha introducido la necesidad de la reestructuración en las formas administrativas, de gerencia y gestión de la organización.

Ahora bien, el caso de JAB S.A.S surge por la necesidad de la empresa de generar nuevos 
productos para involucrarse en un mercado más amplio, es decir efectuar una mayor oferta. Aunque todos los casos son diferentes a cada uno le es transversal el interés financiero, pues el fin de cada empresa en generar mayores ingresos y por tanto mayor estabilidad en el mercado. En este sentido Fernández (2001) menciona que la necesidad de implantar el modelo del BSC en una empresa depende independientemente de su tipo, es decir independientemente de si es grande, mediana o pequeña, de los problemas que enfrenta. El BSC es extremadamente útil para aquellas empresas que tengan la necesidad de comunicar la estrategia, aumentar elementos informativos y evaluar los proyectos. También puede consistir en un elemento de ayuda de planificación, o en un elemento de mecanismo de información y seguimiento de actividades de gestión. Independientemente el caso el BSC, es ese enlace entre la gestión y la planificación. El modelo del BSC varía de acuerdo a las necesidades de cada compañía y su estructura se puede establecer en base a una infinidad de variables. En las VI jornadas académico profesionales de BSC. (2008) se encuentran múltiples ejemplos de la instauración del BSC en varias partes del mundo.

\section{El BSC y su implementación en empresas en crisis organizacional}

Como se observó anteriormente, la implementación del BSC varía de acuerdo a las necesidades de una empresa. En este segmento se analizan las razones por las que una empresa se encuentra en crisis y se comparan estos factores con el modelo del BSC. Esto se efectúa con el objetivo de mostrar los avances, transformaciones y los beneficios concretos del Balanced Scorecard, respecto a la gestión organizacional para empresas en crisis.

En esta medida, se realizó una compilación general de datos tomados de las principales revistas (Revista Dinero, Semana y Portafolio durante el periodo 2005-2017) que manejan el tema de economía en el país. Estas revistas ofrecen un panorama general del por qué una empresa entra en crisis e inclusive llegan a caer en la liquidación. Se puede observar que los factores señalados por las revistas se ubican dentro de las 4 dimensiones que maneja el BSC. (Eje financiero, de clientes, procesos internos y procesos de aprendizaje y desarrollo), A continuación, se realiza una síntesis de la recopilación de datos y su clasificación según perspectiva de BSC.

Ahora bien, como se pudo observar en el Tabla 3 , no todos los factores de crisis en una empresa pueden ser observados por el BSC, uno de estos es el de los fenómenos naturales, puesto que son factores externos que se salen del control del ser humano. Los fenómenos naturales pueden ser impredecibles, pero así mismo hay estadísticas que calculan los porcentajes en los que estos pueden incidir en un territorio determinado, así que también pueden llegar a ser predecibles a un largo plazo. Sin embargo, son aspectos que no se tienen presentes en el momento de realizar el modelo del BSC, por tanto, analizar este aspecto esta por fuera de los límites de esta investigación.

La revista Estrategia y Negocios (2014) señala los siguientes aspectos de una empresa en crisis: Una empresa se topa con una situación de crisis usualmente por la combinación de múltiples factores que interactúan negativamente entre ellos, factores internos y externos, los cuales se pueden resumir en cuatro grandes dimensiones (1) Falta de liquidez y crisis de capital de trabajo, (2) Inhabilidad para cumplir con obligaciones financieras 0 de mantener indicadores financieros razonables, (3) Industria o sector económico amenazado por fuerzas externas imposibles de resolver y (4) Temas laborales o gerenciales que provocan un organización disfuncional. 
Tabla 3. Principales características para que una empresa entre en crisis

Table 3. Main reasons behind a corporate crisis

\begin{tabular}{l|l|l}
\hline \multicolumn{2}{c}{ Principales características para que una empresa entre en crisis y comparación con las } \\
perspectivas del BSC
\end{tabular}

Fuente: elaboración propia 2017.

El último estudio realizado por la Cámara de Comercio (2009) señala factores mucho más específicos por los que una empresa entra en crisis. De esta forma en el 2008 se realizó una investigación con 579 empresas que pasaron por un proceso de liquidación entre el año 2004 al 2008. En esta investigación se pudo observar que el $29 \%$ de las empresas cerraron por pocas ventas, pocas ganancias o porque financieramente generaban solo pérdidas. El $18 \%$ atribuía su liquidación a la falta de recursos económicos para inyectar en su empresa. El $13 \%$ especificó una falta de acuerdo entre socios, lo que afecta directamente el clima organizacional. El $12 \%$ describió la falta de motivación, lo que genera desilusión y por tanto cansancio por el negocio.
También el $11 \%$ de las empresas describió que su fracaso estuvo inmerso en problemas externos a la organización, es decir problemas en el mercado o actividad financiera. El $11 \%$ de las empresas explicó que su liquidación fue ocasionada por la competencia desleal y el contrabando, también la repercusión de actividades ilícitas de extorción, robos, estafas amenazas, entre otros. El $2 \%$ afirmó que la empresa cerró por problemas con el personal. El $9 \%$ indicó que la razón para cerrar la compañía se debió al pago de impuestos o al vencimiento de la licencia y los permisos legales. Y el $28 \%$ describió otros problemas, como las enfermedades, problemas personales, el cambio de razón social o tipo de sociedad, el cambio de actividad y contratación en una empresa, entre otros. Lo interesante de este resultado es que solo el $4 \%$ de las 
empresas atribuyó como una razón principal de cierre la inexperiencia o la falta de conocimiento para manejar una empresa.

Aquí vale la pena señalar una digresión en cuanto a este $4 \%$, puesto que esto nos indica la falta de conciencia de varios empresarios respecto a lo que significa la experiencia y el conocimiento para manejar una empresa, que sobre todo en este país es empírico. Según la revista Dinero (2015) la mayoría de empresas pequeñas fallan en la poca importancia que le dan a los estudios para generar a la vez mecanismos de innovación, que ayuden a una empresa a posicionarse y adaptar al mercado en la época de globalización. En donde finalmente es imperativo que las empresas tengan un direccionamiento estratégico.

Adicional es importante tener en cuenta la ciencia y la tecnología en la organización como lo menciona Becerra-Arevalo, Y. M (2015) es vital tener un balance de la implementación del Fondo de Ciencia, Tecnología e Innovación del Sistema General de Regalías en Colombia (FCTel-SGR). La entrada en vigencia del SGR y la respectiva creación del FCTel-SGR, ha abierto una oportunidad para la ciencia, la tecnología y la innovación en el país y se espera que estos nuevos recursos impacten a las regiones. Antioquia se destaca por el número de proyectos aprobados y la diversificación de temas estratégicos priorizados para presentar al FCTel-SGR, enfocando los esfuerzos en el fortalecimiento de la CTel en los sectores de la salud, agropecuario y formación de capital humano, entre otros.

Retomando el punto anterior, es posible identificar que los motivos se encuentran en su mayoría solucionadas por el BSC, o son características que se tocan dentro del modelo. Al observar la Tabla 3 y la investigación de la cámara de comercio se puede ubicar que la mayoría de las razones por las que una empresa entra en crisis puede ser trabajada directamente por alguno de las 4 perspectivas que maneja el BSC, bien sea financiera, en relación al cliente, en relación a aspectos internos $\mathrm{o}$ a procesos de aprendizaje $\mathrm{y}$ desarrollo.

En este sentido, se logra observar la utilidad que puede tener la implementación del BSC en una empresa en crisis, pues desde unos indicadores medibles, proporcionar las herramientas necesarias para posicionar a una empresa y a la vez a partir de diversas estrategias, funciona como un amortiguador para las épocas de crisis. Para implementar de forma adecuada el BSC, como se ha visto en el desarrollo del documento, resulta fundamental comprender las necesidades de la empresa, y si es una empresa en crisis tener una conciencia de ¿Por qué la organización está en crisis, ¿cuáles son las razones? A partir de esta pregunta se consolida una base para el desarrollo de la implementación del BSC, dentro de los procesos internos de la compañía.

\section{CONCLUSIONES Y RECOMENDACIONES: HACIA UNA IMPLEMENTACIÓN EXITOSA DEL BSC}

Se realizó un estudio sobre las principales causas por las que una empresa entra en crisis y se resaltó la importancia de observar que cada caso en cada empresa es único, por lo que su instauración debe realizarse de una manera contextual, es decir, directamente frente a la organización y su contexto económico y social. De esta forma a partir del recorrido histórico, la observación de los tres ejemplos y la recopilación y análisis de documentos sobre la implementación exitosa del modelo, a continuación, se responde a la pregunta central de la investigación ¿De qué manera el BSC posibilita una oportuna gestión en empresas con crisis organizacional? En consecuencia, en esta sección se realiza énfasis en las empresas en crisis y las formas en que el BSC puede verse como una herramienta útil para lograr generar un fortalecimiento en las estructuras organizacionales y gerenciales de una empresa. 
En primer lugar, es importante aclarar que si bien el BSC es una herramienta que puede ser de utilidad para las empresas en crisis, no es una herramienta mágica que saque a una organización de su posible liquidación, puesto que en este artículo se expone más como una herramienta de control, autoconocimiento, seguimiento y evaluación.

En segundo lugar, hay múltiples razones por las que una empresa puede llegar a presentar un estado de crisis, como se expusieron anteriormente algunos elementos o factores son abordados mediante la herramienta del tablero de mando balanceado. Si bien los ejemplos anteriormente expuestos obedecen a compañías en diversas circunstancias, es importante resaltar que en cada uno de los ejemplos hay puntos de conexión como puntos totalmente aparte.

Así mismo, cabe señalar que usualmente las crisis surgen a partir de características las cuales las empresas tienen conciencia. Es decir que cuando hay crisis una empresa ya intuye la razón, pues en efecto usualmente estos eventos se califican como procesos que surgen paulatinamente, rara vez una crisis surge de un solo golpe.

Hay crisis imposibles de diagnosticar y que escapan a cualquier nivel razonable de anticipación, pero la estadística muestra que la enorme mayoría de casos de crisis ocurren respecto de cosas en las que la organización es absolutamente consciente de que pueden ocurrir (Remy, 2013, p. 4).

En común con los ejemplos anteriormente señalados de las tres empresas, encontramos que la metodología aplicada para los tres casos obedece a una serie de fases que corresponden a una ubicación contextual, para crear el BSC por medio de la construcción de las 4 dimensiones. Esto se realiza a través de la construcción de un mapa estratégico y después se contrasta con una tabla de control, en la cual van inmersos los objetivos, los indicadores y las estrategias. En los tres ejemplos se puede establecer que las empresas mejoran sus redes de comunicación, así como la inclusión y participación de los integrantes, hasta llegar a un acuerdo entre las partes. Por lo que se rescatan aspectos claves como el papel del liderazgo y la disciplina para llevar a cabo los procesos descritos en el BSC, independientemente el caso.

A la vez, a partir del estudio de los tres casos, se ubicó que el BSC no es una herramienta que se aplica y se construye solo una vez, sino que está en constante articulación con los procesos de la compañía y por lo tanto no es un tablero de mando estático sino dinámico. Esta característica de versatilidad, surge con el objetivo de adecuar la empresa a los nuevos ritmos de la globalización en los que los cambios cada vez se realizan de formas más abruptas. Olarte y García (2009) señalan que si se va a implementar el BSC es necesario que se vuelva una costumbre empresarial, la cual se ubique en un proceso de constante construcción y reconstrucción.

Ahora bien, las empresas que enfrentan una crisis primero deben ubicar el motivo de la crisis y comparar con los fundamentos del BSC, figura 1, para ver si esta herramienta es de utilidad en el momento de la crisis, claramente como herramienta de gestión empresarial lo ideal es que estuviera aplicado en la empresa independientemente el estado en que se encuentre, es decir si es de éxito o es de crisis. El modelo, resulta de gran importancia para la implementación en empresas que se encuentran en crisis organizacional, bien sea por los casos anteriormente expuestos, es decir por cambios del mercado, competencia, crecimiento de una compañía. O porque los modelos de gestión no otorgan resultados satisfactorios para una compañía. 


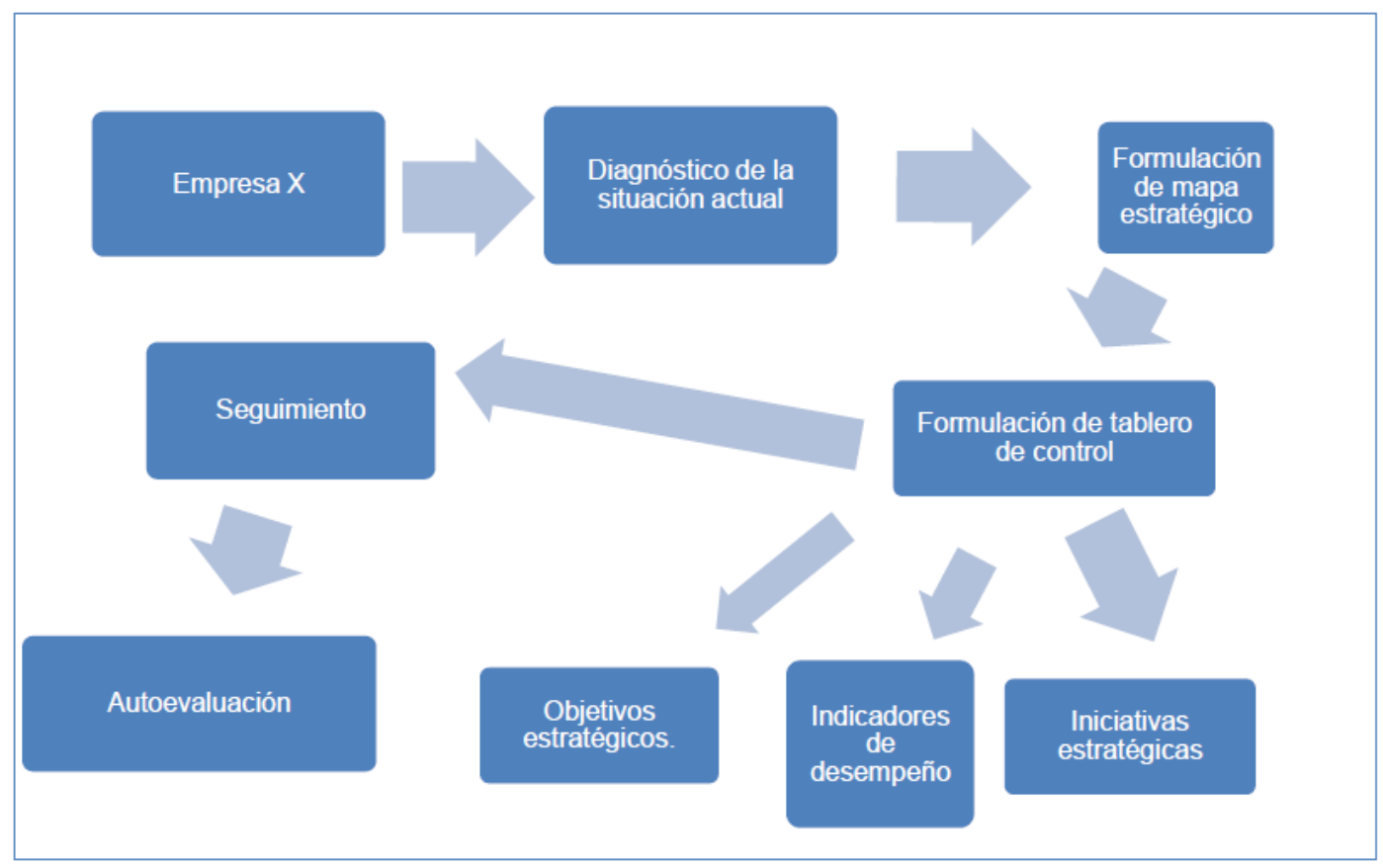

Figura 1. Esquema de similitudes aplicación de BSC de 3 empresas

Figure 1. Scheme of similarities in the application of the BSC at three companies Fuente: Elaboración propia, 2017.

Para estudiar los beneficios concretos del Balanced Scorecard, respecto a la gestión organizacional para empresas en crisis, en la investigación documental se realizó una serie de aproximaciones a autores que describen factores de éxito para la implementación del tablero de mando balanceado. Entre estos estos Olarte y García (2009), describen que las empresas se enfrentan a una serie de obstáculos cuando intentan implementar el modelo del BSC, como el paso del papel a la realidad, es decir la ejecución de la estrategias, la comunicación de estas estrategias hacia los demás departamentos de las empresas, la falta de dedicación de tiempo para la discusión de las estrategias, la falta de vincular las partes financieras de una empresa con las estrategias, y la falta de formular incentivos de parte de los departamentos de gerencia que se vinculen con las estrategias establecidas en el BSC.

Según Kaplan Y Norton (2001), se estima que el $50 \%$ de las empresas que afirma estar implementando el modelo de BSC en su organización, lo realizan de una forma errónea u incompleta. Esto se debe enfrentar a diversas dificultades que corresponden a que la empresa no logre adaptarse al cambio, lo que imposibilita un proceso de transición a una cultura del BSC, lo anterior se da porque no se da continuidad a la implementación de diferentes estrategias en la organización. Así mismo, los diseños que se acogen en la implementación del modelo de BSC para una determinada empresa, no cubren con éxito las demandas de ésta. Otra dificultad en su implementación, está ligada a la falta de compromiso por parte de los responsables y por parte del personal de la empresa.

En este sentido, para garantizar el éxito en la implementación del modelo del BSC hay que ubicar dimensiones clave en la instauración. A partir de la recopilación y el análisis de diferentes documentos, se logró identificar que es clave el papel de la figura del líder, junto con el apoyo directivo. Como se señaló en los anteriores ejemplos y en el cuerpo del artículo es importante que en cada departamento la figura de líder, corresponda a las exigencias demandadas por el BSC, es decir que debe ser una persona pro activa, que incentive a su 
departamento de acuerdo a el cumplimiento de una serie de estrategias vinculadas al BSC. Así mismo la figura de líder es clave para gestionar una comunicación interna efectiva entre los departamentos y los directivos.

Esto se señala para una empresa grande, mediana y también aplica en empresas pequeñas, la figura del líder debe ser esa persona capaz de generar una integración o inclusión efectiva entre la empresa con sus empleados, mejorando las redes de comunicación.

Otra dimensión clave, consiste en observar y justificar si se necesita el tablero de mando balanceado en una empresa, para así lograr asegurar que se use el BSC de una forma continua y efectiva. Para planear el proyecto de BSC, se dimensiona la formulación de preguntas guía ¿Qué? ¿Por qué? ¿Para qué? ¿Cómo? ¿Cuándo? ¿En dónde? ¿Quién?

Así mismo resulta importante validar las hipótesis creadas por el BSC en la empresa, puesto que el BSC se maneja mediante la fórmula de la causa y el efecto (Malgioglio et al., 2002), por lo que corroborar su estructura diseñada en los departamentos de una empresa representa el éxito de su implementación. Para realizar esta corroboración usualmente se utilizan estudios de correlación estadística, pues son estos los que nos indican la fuerza de la relación entre las variables cualitativas y cuantitativas que maneja el BSC.

A partir de lo anterior se deben definir las iniciativas estratégicas, el cual se realiza después de la definición de fines, para responder cómo se van a alcanzar esas metas. También, resulta importante darles un orden a estas iniciativas ubicándolas por prioridad y así formular unas líneas clave de acción, para optimizar los resultados de la organización.
Cuando se formula este plan estratégico, es importante entender que, así como la figura del líder es clave, la actuación de los empleados también lo es pues depende de la motivación y compromiso que estos tengan con la empresa alcanzar los resultados y las metas. Para lograr una inclusión efectiva de cada integrante de la organización, en el desarrollo de la empresa es fundamental la comunicación y la constante capacitación o también, la implementación de procesos educativos que la empresa tiene sobre sus empleados.

Ahora bien, la educación sobre el BSC en la empresa puede realizarse de diversas maneras, sin embargo, deben ser procesos continuos y consistentes, también pedagógicos. Entendiendo que los apoyos visuales e interactivos a la vez mejoran el clima organizacional, y a partir de una estimulación positiva en los empleados de la empresa, se puede lograr generar una inclusión y comunicación efectiva. Cabe resaltar, que Olarte y García (2009) describen que el proceso del BSC comienza por los altos cargos y la dirección, pero finalmente es un proceso que se debe interiorizar en cada uno de los integrantes de la empresa independientemente del cargo. Crear esta malla de inclusión del personal en todos los niveles, sin duda generará una comunicación más asertiva, logrando el cumplimiento efectivo de actividades para llegar a cumplir metas y objetivos.

Una de las formas en las cuales se consigue motivar al personal, es a través de la formulación de incentivos por los resultados de sus trabajos y el cumplimiento de las metas, las cuales a su vez deben ser metas objetivas, claras y alcanzables, asegurándose de realizar estos procesos con la mayor transparencia y honestidad posible frente a sus empleados. Es decir, que estos incentivos deben realizarse a través de un trabajo medible y un principio de justicia, entre los integrantes de una organización a través de la equidad. Por medio de la compresión de las diferencias entre cada 
departamento, cada cargo e inclusive entre cada trabajador, se deben establecer los incentivos.

En este sentido, cuando se crea un ambiente organizacional pleno, también se genera una conciencia en cuanto a las formas de competencia, diferenciando lo que es la competencia desleal, frente a la competencia sana. Cuando se aplica un principio de competencia sana, se ayuda a generar superación personal por medio de diversos mecanismos de apoyo, este es un tema debatible en diversos ámbitos de las ciencias sociales. Pues el concepto de competencia aún está en construcción en la academia y parte desde diversas perspectivas. Sin embargo, este artículo pretende reconocer algunos factores para que las empresas en crisis organizacional salgan adelante a través del BSC, por lo que su debate se ubica por fuera de los límites del documento. Aun así, se recomienda que la empresa ubique la definición de competencia que más se adecué a su contexto, y esta definición se inculque en cada empleado.

Por otro lado, la inserción de la tecnología dentro de los procesos de la compañía es un factor fundamental de éxito, pues en la era de la globalización cada vez el uso de la tecnología es más una necesidad que un lujo. En este caso se ubicó la posibilidad de hacer uso de la tecnología para el establecimiento y creación del BSC, hoy en día hay varios softwares que ofrecen modelos para aplicar el tablero de mando balanceado.

\section{CONCLUSIONES}

A partir de los resultados obtenidos y descritos anteriormente se aprueba y corrobora la hipótesis de la que parte esta investigación, es decir que el BSC es de suma utilidad y su implementación tiene un efecto positivo y necesario dentro de las empresas en crisis. En consecuencia, después de analizar el recorrido histórico, fue posible entender que el BSC es una herramienta que surge precisamente para lograr adaptar a las empresas a los nuevos cambios que experimenta el mercado en la época de la globalización y a partir de la implementación del modelo económico liberal. En este sentido se concluye que el BSC es una herramienta que no es un lujo sino inclusive llega a ser una necesidad para cualquier empresa. Dado que se creó con la intención de ser una herramienta útil y de ayuda para la prosperidad y sostenibilidad de una empresa.

Por otro lado, se observa que la crisis organizacional es un proceso del cual ninguna empresa está exenta, y de hecho es muy probable que una compañía deba pasar por estos procesos de crisis. Por lo que se concluye que el BSC es una herramienta que debe ampliarse hasta que logre formular también un método de anticipación y prevención. En consecuencia, el BSC es una herramienta que permite a partir de la conciencia de los procesos internos de una organización, la formulación de posibles riesgos y por tanto la recuperación ante las crisis. Según las estadísticas cuando una empresa tiene un sistema de prevención, se generan una mitigación de las dificultades de una forma más rápida y efectiva según Remy (2013). Es decir que cuando se logra conocer a fondo el diseño y organización de una empresa es más fácil generar un mapa de riesgos como elemento que complemente el BSC.

EI BSC es un proceso que varía de acuerdo a la necesidad de una empresa, por lo tanto, para su instauración es fundamental tener claro el contexto histórico de la compañía y la visión ¿A dónde quiere llegar?, ya que el BSC se establece a partir de la necesidad. Cada caso es único, cada empresa es única, sin embargo, hay un modelo que aplica para todas las empresas independientemente su estructura, es decir su tamaño. Así mismo, aunque todas las empresas son diferentes el eje de crecimiento financiero es transversal a todas las compañías, por lo que, a partir de las similitudes, es un modelo 
que indiscriminadamente se puede aplicar a cualquier negocio.

Al ultimar la investigación se logró observar que el BSC, como herramienta de aprendizaje e inclusión que potencializada la unión para el trabajo en equipo, si bien el BSC no es una herramienta para que una empresa supere la crisis en sus diversas formas, si es un instrumento que mejora de muchas maneras la estructura en la que se gerencia y planifica una compañía y sirve para amortiguar los procesos de crisis y predecirlos, por lo que es de gran utilidad en un periodo de crisis.

Es por esto que finalmente las empresas que enfrentan crisis organizacional, pueden observar en esta herramienta una solución para la forma en que se está construyendo desde su interior y la influencia del exterior. El BSC ayuda a generar un mayor conocimiento respecto a la compañía, y a través de ese conocimiento logra fomentar la estructuración de redes comunicativas, que permiten generar una unión en los diversos departamentos de la empresa.

\section{REFERENCIAS}

Alcaldía de Bogotá. (2007). Guía Práctica para la elaboración del Cuadro de Mando Integral BSC. Recuperado de https://www.yumpu.com/es/document/v iew/16394279/cuadro-de-mandointegrado-bsc-alcaldia-mayor-de-bogota

Amaru, C. (2009). Fundamentos de administración. Teoría general y proceso administrativo. México. Pearson Educación.

Armijo, M. (2009). Manual de Planificación Estratégica e Indicadores de Desempeño en el Sector Público. ILPES/CEPAL. Recuperado de http://www.cepal.org/ilpes/noticias/pagi nas/3/38453/manual_planificacion_estra tegica.pdf

Becerra-Arévalo, Y. M. (2015). Sistema general de regalías: nuevos recursos para la ciencia, tecnología e innovación en Colombia. Revista CEA, 1(1), 75-91. http://itmojs.itm.edu.co/index.php/revist a-cea/issue/view/55

Cámara De Comercio (2009) Informe sobre las Causas de la liquidación de empresas en Bogotá. Recuperado de http://bibliotecadigital.ccb.org.co/bitstre am/handle/11520/13127/4439_causas_d e_liquidacion_de_empresas_en_bogota_ parte_1-1.pdf?sequence=1\&isAllowed=y

Coombs, W. (2014) Crisis Management and Communications. Institute for public relations. Recuperado de http://www.instituteforpr.org/crisismanagement-communications/

Creación de empresas en Colombia aumentó un $17 \%$ en el primer semestre del año. (2016 21 de julio). Portafolio. Recuperado de http://www.portafolio.co/negocios/aum enta-la-creacion-de-empresas-encolombia-498992

Fernández, A. (2001). El Balanced Scorecard: Ayudando a implantar la estrategia. IESE, Revista de antiguos alumnos. pág. 31-42. Recuperado de http://planuba.orientaronline.com.ar/wp -content/uploads/2009/10/20belbalanced-scorecard-ayudando-aimplantar-la-estrategia-iese.pdf

García, J., \& Olarte, A. (2009). Factores clave de éxito para una implantación exitosa del sistema de Gestión estratégica "Balanced Scorecard". Revista EAN, 65, 49-76. Recuperado de http://journal.ean.edu.co/index.php/Revi sta/article/viewFile/460/451 
Grajales, J. y Maldonado, L. (2015) diseño del Balanced Scorecard (bsc) para la empresa Laminados JAB S.A.S. Tesis de pregrado. Universidad Militar Nueva Granada. Bogotá

Kaplan, R. \& Norton, D. (2008). El Cuadro de Mando Integral. Madrid, España: Editorial Gestión 2000.

¿Por qué fracasan las pymes en Colombia? (2015, 9 de febrero) revista Dinero. Recuperado de http://www.dinero.com/economia/articu lo/pymes-colombia/212958

Malgioglio, J. et al., (2002). Distintos enfoques del capital intelectual. Séptimas Jornadas "Investigaciones en la Facultad" de Ciencias Económicas y Estadística.

Mañas, L. D. (2006). El Cuadro de Mando Integral (BSC), gestionar eficientemente la visión. Escuela Superior de Negocios y Tecnologías. Recuperado de https://navactiva2013.wordpress.com/20 06/01/17/el-cuadro-de-mandointegral bsc -gestionar-eficientemente-lavi_23217/

Más allá de los indicadores (2005, 15 de octubre). Revista Dinero. Recuperado de http://www.dinero.com/edicionimpresa/management/articulo/mas-allaindicadores/30516

Muerte de empresas superó a la creación (2015, 22 de enero). Portafolio. Recuperado de http://www.portafolio.co/economia/fina nzas/muerte-empresas-supero-creacion25160

Neriz, L. et al., (2006). El proceso de cascada del cuadro de mando Integral en empresa del sector forestal. Chile: Universidad de Talca.
Norton, D. Kaplan, R. (2014). El cuadro de mando integral: The balanced scorecard. España. Grupo Planeta.

Ocho causas por las que empresas entran en crisis (2017). Finanzas personales. Revista Semana. Recuperado de http://www.finanzaspersonales.com.co/a horro-e-inversion/articulo/ocho-causasempresas-entran-crisis/51066

Ortiz-Delgadillo, G., Esquivel-Aguilar, E. O., \& Hernández-Castorena, O. (2016). El impacto de la relación con el cliente y de la capacidad de valor agregado en el servicio en el rendimiento de la Pyme Manufacturera en Aguascalientes. Revista CEA, 2(4), 47-58. http://revistas.itm.edu.co/index.php/revi sta-cea/article/view/171

Remy, P. (2013). Manejo Estratégico de crisis en organizaciones: cómo preverlas, enfrentarlas y aprender de ellas. Sinergia e Innovación, 1(08). Recuperado de http://repositorioacademico.upc.edu.pe/ upc/bitstream/10757/332956/1/92-3081-PB.pdf

Schvartzman, V., \& Vogel, M. (2011) Gestión por competencias y su articulación con el tablero de comando. Cooperar. Recuperado de http://api.ning.com/files/BVZ82dL9OH-gsl-e7e8-IxjLGrUdrN9BbzY*s*VuqsEFylfwQSucTGaLFFeXZcfmBpRFoXz6US1Q4ReHw AtXV0e*v1aeX/ArticuloArticulacionComp etenciasyTablerodeComando.pdf

Trullenque, F. Linquete, J. (2002). El modelo integrado $\mathrm{EFQM} \& \mathrm{BSC}$ transformando estrategia en acción excelente. Revista Harvard Deusto Finanzas y Contabilidad, 46, marzo - abril, 28-39. 